Pacific Journal of Mathematic 


\title{
HOMOTOPY GROUPS OF PL-EMBEDDING SPACES
}

\author{
L. S. HuSCH
}

Let $N$ be a compact $P L-n$-manifold, and let $M$ be a $P L$ $m$-manifold without boundary. Two of the major problems in $P L$-topology are to determine conditions such that $(1)$ any continuous map of $N$ into $M$ can be homotoped to a $P L$ embedding, and (2) two homotopic $P L$-embeddings are $P L$ isotopic.

If $C(N, M)$ is the space of continuous maps of $N$ into $M$ with the compact open topology, and if $P L(N, M)$ is the subspace of $P L$-embeddings, one can consider the map $i_{\sharp:} \Pi_{0}(P L(N, M)) \rightarrow \Pi_{0}(C(N, M))$ induced by inclusion. If $(\mathbf{1})$ is true, then $i_{\#}$ is onto; if $(2)$ is true, then $i_{\#}$ is one-to-one. In this paper, we investigate the higher homotopy groups of $P L(N, M)$ and $C(N, M)$.

Irwin has shown that if $N$ is a closed manifold, $m \geqq n+3$, then sufficient conditions for (1) are that $N$ is $(2 n-m)$-connected and $M$ is $(2 n-m+1)$-connected. By raising the connectivities of $N$ and $M$ by one, Zeeman [7] proved (2).

By using Proposition 1 of Morlet [4] and Irwin [3], one can easily show the following theorem by using techniques similar to the proof of Theorem 2 below.

Theorem 1. Let $N$ be a closed $(2 n+s+1-m)$-connected $P L$ $n$-manifold and let $M$ be a $(2 n+s+2-m)$-connected PL-mmanifold without boundary, $m \geqq n+3$. The homomorphism $i_{\sharp}$ : $\Pi_{s}(P L(N, M)) \rightarrow \Pi_{s}(C(N, M))$ induced by inclusion is an isomorphism; if the connectivities of $N$ and $M$ are lowered by one, then $i_{\#}$ is onto.

An analogous theorem in the differential case has been proved by J. P. Dax [1], [2].

If $N$ has a nonempty boundary, then Dancis, Hudson and Tindell (independently and unpublished) have shown that if $N$ has a $k$ dimensional spine with $m \geqq\{n+3, n+k\}$, this is a sufficient condition for (1). If $m \geqq\{n+3, n+k+1\}$, they obtain (2). We generalize.

THeORem 2. Let $N$ be a compact PL-n-manifold with $k$-spine $K$, $k<n$, and let $M$ be a PL-m-manifold without boundary. If $m \geqq n+k+s+1$, the homomorphism $i_{\sharp}: \Pi_{s}(P L(N, M)) \rightarrow \Pi_{s}(C(N, M))$ induced by inclusion is an isomorphism; if $m \geqq n+k+s$, $i_{\#}$ is onto.

Note that the codimension 3 restriction is eliminated. In $\S 3$, 
we obtain some consequences of this theorem and its proof.

The author wishes to express his gratitude to N. Max who read a preliminary version of this paper and suggested some corrections.

In this paper, we shall consider $P L(N, M)$ and $C(C, M)$ as 4 -sets (-i.e., as semisimplicial complexes in which the degeneracy maps are ignored). In $\S 1$, we list the basic definitions and results on $\Delta$-sets which we shall use. One may use either Rourke and Sanderson [6] or Morlet [5]. [Morlet uses the terminology "quasisimplicial" set.]

We shall assume familiarity with either [1] or [7] and shall use terminology therein with one exception. When referring to piecewise linear maps or manifolds, we shall always use the prefix " $P L$-".

Let $X$ and $Y$ be polyhedra. In this paper $p_{1}$ and $p_{2}$ will always denote projections of $X \times Y$ onto the first and second factors respectively. An isotopy between $X$ and $Y$ will be represented as a family of embeddings $f_{t}: X \rightarrow Y, t \in I=[0,1]$.

1. $\Delta$-sets. Let $\Delta^{n}$ denote the standard $n$-simplex with ordered vertices $v_{0}, v_{1}, \cdots, v_{n}$. The $i$-th face map $\partial_{i}: \Delta^{n-1} \rightarrow \Delta^{n}$ is the order preserving simplicial embedding which omits $v_{i} . \Delta$ is the category whose objects are $\Delta^{n}, n=0,1, \cdots$ and whose morphisms are generated by the face maps. A $\Delta$-set $(\Delta-g r o u p)$ is a contravariant functor from $\Delta$ to the category of sets (groups). A $\Delta$-map between $\Delta$-sets ( $\Delta$-groups) is a natural transformation between the functors.

If $X$ is a $\Delta$-set, $X^{k}=X\left(\Delta^{k}\right)$ is the set of $k$-simplexes and the maps $\partial_{i}=X\left(\hat{o}_{i}\right)$ are called face maps. We shall be interested in pointed $\Delta$-sets in which we distinguish a simplex $*^{k} \in X^{k}$ for each $k$ and designate $* \subset X$ as the sub- $\Delta$-set of $X$ consisting of these simplexes and maps $\partial_{i}$ defined by $\partial_{i} *^{k}=*^{-1}$.

With each ordered simplicial complex $K$, we associate a $\Delta$-set, also designated by $K$, whose $k$-simplexes are order-preserving simplicial embeddings of $\Delta^{k}$ into $K$.

Let $A_{n, i}=\mathrm{Cl}$ (bdry $\left.\Delta^{n}-\partial_{i} \Delta^{n-1}\right)$. A $\Delta$-set $X$ is called a Kan $\Delta$-set if every $\Delta$-map $f: \Lambda_{n, i} \rightarrow X$ can be extended to a $\Delta-\operatorname{map} f_{1}: \Delta^{n} \rightarrow X$.

If $X$ is a Kan $\Delta$-set and $P$ is a polyhedron, a map $f: P \rightarrow X$ is a $\Delta$-map $f: K \rightarrow X$ where $K$ is an ordered triangulation of $P . f_{0}, f_{1}: P \rightarrow X$ are homotopic if there is a map $F: P \times I \rightarrow X$ such that $F \mid P \times\{i\}=$ $f_{i}, i=0,1$. $[P ; X]$ denotes the set of homotopy classes. We shall need the following two propositions which are proved by Rourke and Sanderson.

Proposition 1. Any homotopy class in $[P ; X]$ is represented by a $\Delta$-map $f: K \rightarrow X$ where $K$ is any ordered triangulation of $P$.

Proposition 2. Let $Q$ be a subpolyhedron of $P$ and let 
$h: Q \times I \cup P \times\{0\} \rightarrow X$ be a $\Delta$-map to a Kan $\Delta$-set $X$; then $h$ extends to a $\Delta$-map $h^{\prime}: P \times I \rightarrow X$.

If $X$ is a pointed Kan 4 -set, then the $n$-th homotopy group of $X, \Pi_{n} X=\left[I^{n}\right.$, bdry $\left.I^{n} ; X, *\right]$, the homotopy classes of $\Delta$-maps of pairs, where $I^{n}$ is the $P L$ - $n$-cell.

$C(N, M)(P L(N, M))$ is made into a $\Delta$-set by defining the $k$ simplexes to be maps ( $P L$-embeddings) $f: N \times \Delta^{k} \rightarrow M \times \Delta^{k}$ such that $p_{2} f=p_{2}$ and defining $\partial_{i} f=f \mid N \times \partial_{i} \Delta^{k}$.

Proposition 3. $C(N, M)$ and $P L(N, M)$ are Kan 4 -sets.

Proof. Let $f: \Lambda_{n, i} \rightarrow P L(N, M)$ be a $\Delta$-map. $f$ can then be considered as a $P L$-embedding

$$
f: N \times \Lambda_{n, i} \longrightarrow M \times \Lambda_{n, i}
$$

such that $p_{2} f=p_{2}$. Using the fact that the pair $\left(\Lambda_{n, i} \times I, \Lambda_{n, i} \times\{0\}\right)$ is $P L$-homeomorphic to $\left(\Delta^{n}, A_{n, i}\right)$, one can easily construct the desired extension.

2. Proof of Theorem 1. The following two propositions are generalizations to product spaces of the simplicial approximation and general position theorems. They can be proved similarly.

Proposition 4. Let $M$ and $Y$ be $P L$-manifolds and let $P \subseteq Q$ be compact polyhedra. Suppose $f: Q \rightarrow M \times Y$ is a continuous map such that $f \mid P$ is $P L$. There exists a homotopy $h_{t}: M \times Y \rightarrow M \times Y$, $t \in I$, such that
(i ) $p_{2} h_{t}=p_{2}$ for $t \in I$;
(ii) $h_{t} f \mid P=f$ for $t \in I$;
(iii) $h_{1} f: Q \rightarrow M \times Y$ is $P L$.

Proposition 5. Let $M$ and $Y$ be $P L$-manifolds and let $P \subseteq Q$ be compact polyhedra. Suppose $f: Q \rightarrow M \times Y$ is a $P L$-map such that $f \mid P$ is a PL-embedding. There exists a PL-homotopy $h_{t}: M \times Y \rightarrow M \times Y, t \in I$, such that

(i ) $p_{2} h_{t}=p_{2}$ for $t \in I$;

(ii) $h_{t} f \mid P=f$ for $t \in I$;

(iii) the singular set of $h_{1} f$ has dimension $\leqq 2 \operatorname{dim} Q-\operatorname{dim}(M \times Y)$;

(iv) the branch set of $h_{1} f$ has dimension $<2 \operatorname{dim} Q-\operatorname{dim}(M \times Y)$.

The following two constructions are needed frequently in the following propositions.

Proposition 6. Let $N$ be a PL-n-manifold with k-spine $K . \quad$ Let 
$P$ be a polyhedron in $N$ such that $\operatorname{dim} P+\operatorname{dim} K+1 \leqq \operatorname{dim} N$. There exists a PL-isotopy $H_{t}$ of $N, t \in I$, such that $H_{0}=i d e n t i t y$ and $H_{1}(N) \cap P=\varnothing$.

Proof. By general position, we can find a $P L$-ambient isotopy $L_{t}$ of $N$ so that $L_{1} K \cap P=\varnothing$. Let $N^{\prime}$ be a regular neighborhood of $L_{1} K$ in $N$ such that $N^{\prime} \cap P=\varnothing$. Note that $L_{1} K$ is also a spine of $N$. Hence, by the uniqueness theorem of regular neighborhoods, there is a $P L$-isotopy $H_{t}$ of $N, t \in I$, such that $H_{0}=$ identity and $H_{1}(N)=N^{\prime}$.

Construction $\alpha$. Let $I_{+}^{s}$ be a $P L$-cell in the interior of $I^{s}$ and let $U$ be a neighborhood of $\mathrm{Cl}\left(I^{s}-I_{+}^{s}\right)$ in $I^{s}$. Let $U_{0}, U_{1}$ be regular neighborhoods of $\mathrm{Cl}\left(I^{s}-I_{+}^{s}\right)$ in $I^{s}$ such that $U_{0} \subseteq$ int $U_{1}$ and $U_{1} \subseteq U$. Let $\varphi: S^{s-1} \times I \rightarrow \mathrm{Cl}\left(U_{1}-U_{0}\right)$ be a $P L$-homeomorphism such that $\varphi\left(S^{s-1} \times\{i\}\right)=\operatorname{bdry} U_{i} \cap \operatorname{int} I^{s}, i=0,1$.

Proposition 7. Let $N, K, M$ be as in Theorem 2 with $m \geqq$ $n+k+s$. Let $f: N \times I^{s} \rightarrow M \times I^{s}$ be a $P L$-map such that $p_{2} f=p_{2}$ and such that there exists a neighborhood $U$ of $\mathrm{Cl}\left(I^{s}-I_{+}^{s}\right)$ such that $f \mid N \times U$ is a PL-embedding, then there exists a PL-homotopy $f_{t}: N \times I^{s} \rightarrow M \times I^{s}$ and a neighborhood $V$ of $\mathrm{Cl}\left(I^{s}-I_{+}^{s}\right)$ in $I^{s}$ such that

(i ) $f_{0}=f, p_{2} f_{t}=p_{2}, t \in I$;

(ii) $f_{t} \mid V=f, t \in I$;

(iii) $f_{1}: N \times I^{s} \rightarrow M \times I^{s}$ is a PL-embedding.

Proof. By Proposition 5, we can assume that the singular set $T$ of $f$ has dimension $\leqq 2(n+s)-(m+s)$, the branch set $B \subset T$ of $f$ has dimension $<2(n+s)-(m+s)$, and that $f \mid K \times I^{s}$ is a $P L$ embedding. By Proposition 6, there is a $P L$-isotopy $H_{t}$ of $N$ such that $H_{0}=$ identity and $H_{1}(N) \cap p_{1} B=\varnothing$. Hence there is no loss of generality in assuming that $f \mid p_{1}^{-1}\left(H_{1}(N)\right) \times I^{s}$ is a $P L$-embedding.

Let $U_{0}, U_{1}$ and $\varphi$ be as in construction $\alpha$. Define $F_{t}: N \times I^{s} \rightarrow$ $N \times I^{s}, t \in I$, by

$$
\begin{array}{ll}
\left(H_{t}(x), y\right) & y \in \mathrm{Cl}\left(I^{s}-U_{1}\right) \\
F_{t}(x, y)=(x, y) & y \in U_{0} \\
\left(H_{t t_{0}}(x), y\right) & y \in \mathrm{Cl}\left(U_{1}-U_{0}\right), y=\varphi\left(y_{0}, t_{0}\right) .
\end{array}
$$

Let $f_{t}=f F_{t}$ and $V=U_{0}$.

The following is the theorem of Dancis, Hudson and Tindell mentioned in the introduction. We include the proof for completeness.

Proposition 8. Let $N, K, M$ be as in Theorem 2 with $m \geqq n+k$. There exists a PL-embedding $f: N \rightarrow M$. 
Proof. Let $f^{\prime}: N \rightarrow M$ be a continuous map and approximate $f^{\prime}$ by a $P L$-map $f^{\prime \prime}$ such that $f^{\prime \prime} / K$ is a $P L$-embedding and $f^{\prime \prime}$ is in general position. Let $B \subset S$ be the branch and singular set of $f^{\prime \prime}$ respectively. By Proposition 6, there is a $P L$-isotopy $H_{t}, t \in I$, of $N$ such that $H_{1}(N) \cap S=S \cap K$. Let $f=f^{\prime \prime} H_{1}$.

REMARK. We shall make $P L(N, M)$ and $C(N, M)$ into pointed $A$-sets by defining the basepoint complex $*$ as follows. Let $*^{s}(x, y)=$ $(f(x), y), x \in N, y \in \Delta^{s}$ where $f$ is defined in Proposition 8. The face operators are defined naturally.

The proof of the following proposition is well known.

Proposition 9. Let $N, M, K$ be as in Theorem 2 with $m \geqq n+k$. Let $g: N \times I^{s} \rightarrow M \times I^{s}$ represent an s-simplex in $P L(N, M)(C(N, M))$ such that

$$
g\left|N \times \operatorname{bdry} I^{s}=*^{s}\right| N \times \operatorname{bdry} I^{s},
$$

$g$ is homotopic rel bdry $I^{s}$ in $P L(N, M)(C(N, M))$ to $g^{\prime}: N \times I^{s} \rightarrow$ $M \times I^{s}$ such that for some neighborhood $U$ of $\mathrm{Cl}\left(I^{s}-I^{s}+\right)$ in $I^{s}$, $g^{\prime}\left|N \times U=*^{s}\right| N \times U$.

Proposition 10. Let $N, M, K$ be as in Theorem 2 with $m \geqq$ $n+k+s+1$ and let $F_{t}: N \times I^{s} \rightarrow M \times I^{s}$ be a PL-homotopy such that

(i) $F_{i}$ are $P L$-embeddings, $i=0,1$;

(ii) $p_{2} F_{t}=p_{2}, t \in I$ :

(iii) there exists a neighborhood $U$ of $\mathrm{Cl}\left(I^{s}-I_{+}^{s}\right)$ in $I^{s}$ such that $F_{t} \mid N \times U=*^{s}$.

Then there exists a PL-isotopy $G_{t}: N \times I_{s} \rightarrow M \times I^{s}$ such that

(i) $G_{i}=F_{i}$ for $i=0,1$;

(ii) $p_{2} G_{t}=p_{2}, t \in I$ :

(iii) there exists a neighborhood $V$ of $\mathrm{Cl}\left(I^{s}-I_{+}^{s}\right)$ in $I^{s}$ such that $G_{t} \mid N \times V=*^{s}$.

Proof. Note that there is no loss of generality in assuming that there is an $\varepsilon>0$ so that $F_{t}$ are $P L$-embeddings, $t \in[0, \varepsilon] \cup[1-\varepsilon, 1]$. However, now this is a restatement of Proposition 7.

The proof of Theorem 2 now follows easily from the above propositions.

3. Applications. One of the immediate consequences of Theorem 2 is a partial generalization of Hudson's "concordance implies isotopy" 
theorem [2]. (See also Proposition 1 of [4].)

Corollary 1. Let $N$ be a compact $P L$-n-manifold with $k$-spine $K, k<n$, and let $M$ be a PL-m-manifold without boundary. Let $f: N \times I^{s} \rightarrow M \times I^{s}$ be a PL-embedding such that $p_{2} f \mid N \times$ bdry $I^{s}=$ $p_{2}$. Then if $m \geqq n+k+s$, there exists a PL-embedding $F: N \times I^{s} \rightarrow$ $M \times I^{s} \quad$ such that $F \mid N \times$ bdry $I^{s}=f$ and $p_{2} F=p_{2}$. If $m \geqq$ $n+k+s+1, f$ and $F$ can be chosen to be isotopic rel $N \times \operatorname{bdry} I^{s}$.

Let $X$ be an $s$-dimensional polyhedron and let $p: E \rightarrow X$ and $q: F \rightarrow X$ be $P L$-fiber bundles with fibers $N$ and $M$ respectively with structure groups Aut $(N)$ and Aut $(M)$ where

(i ) $N$ is a $P L$-n-manifold with $k$-spine, $k<n$;

(ii) $M$ is a $P L-m$-manifold without boundary;

(iii) $\operatorname{Aut}(N)$ and $\operatorname{Aut}(M)$ are the groups of $P L$-automorphisms of $N$ and $M$, respectively.

By triangulating $X$ and by using the propositions above together with induction on the dimension of the simplexes of $X$, one can easily prove the following.

Corollary 2. If $f: E \rightarrow F$ is a continuous bundle map (-i.e., $q f=p)$ and $m \geqq n+k+s$, then $f$ is homotopic through bundles maps to a PL-bundle map which is an embedding of $E$ into $F$. If $m \geqq n+k+s+1$; any two PL-bundle embeddings of $E$ into $F$ are isotopic through bundle maps.

A $P L_{m}$-bundle is a $P L$-bundle $q: F \rightarrow X$ whose fiber is Euclidean $m$-space $R^{m}$ and whose structural group is the $P L$-automorphisms of $R^{m}$ mod the origin.

Corollary 3. Let $N$ be a PL-n-manifold with $k$-spine, $k<n$; let $p: E \rightarrow X^{s}$ be a PL-fiber bundle with $N$ as fiber and Aut $(N)$ as structural group. If $m \geqq n+k+s$, then for any $P L_{m}$-bundle $q: F \rightarrow X$, there exists a PL-bundle map $f: E \rightarrow F$ which is an embedding. If $m \geqq n+k+s+1$, then any such two PL-bundle embeddings are isotopic through bundle maps.

\section{REFERENCES}

1. Jean Pierre Dax, Généralisation des théorèmes de plongement de Haefliger à des familles d' applications dépendant d'un nombre quelconque de paramètres, C. R. Acad. Sci. Paris (A-B) 264 (1967), A499-A502.

2. —_ Étude homotopique de l'espace des plongements d'une n-variété dans une m-variété. C. R. Acad. Sci. Paris (A-B) 267 (1968), A190-A193.

3. J. F. P. Hudson, Piecewise linear topology, University of Chicago Mathematics 
Lecture Notes, 1966/67.

4. - Concordance and isotopy of $P L$ embeddings, Bull. Amer. Math Soc. 72 (1966), 534-535.

5. M. C. Irwin, Embeddings of polyhedral manifolds, Ann. of Math. 82 (1965), 1-14.

6. C. Morlet, Les méthodes de la topologie différentielle dans l'étude des variétés semilinéaires, Ann. scient. Éc. Norm. Sup. (4) 1 (1968), 313-394.

7. C. P. Rourke, and R. J. Sanderson, On the homotopy theory of $\Delta$-sets (to appear)

8. E. C. Zeeman, Seminar on combinatorial topology, Inst. des Hautes Études Sci., Paris, 1963.

Received April 11, 1969. Research supported in part by National Science Foundation Grant GP-8615.

UNIVERSITY OF GEORGIA

Athens, Georgia

Virginia Polytechnic Institute

BLACKSBURG, VIRGINIA 



\section{PACIFIC JOURNAL OF MATHEMATICS}

\section{EDITORS}

H. SAMELSON

Stanford University

Stanford, California 94305

\section{RichaRd PIERCe}

University of Washington

Seattle, Washington 98105
J. DUGUNDJI

Department of Mathematics

University of Southern California

Los Angeles, California 90007

RICHARD ARENS

University of California

Los Angeles, California 90024

\section{ASSOCIATE EDITORS}

E. F. BECKENBACH

B. H. NeUManN

F. WOLF

K. YosHIDA

\section{SUPPORTING INSTITUTIONS}

UNIVERSITY OF BRITISH COLUMBIA CALIFORNIA INSTITUTE OF TECHNOLOGY UNIVERSITY OF CALIFORNIA MONTANA STATE UNIVERSITY

UNIVERSITY OF NEVADA

NEW MEXICO STATE UNIVERSITY

OREGON STATE UNIVERSITY

UNIVERSITY OF OREGON

OSAKA UNIVERSITY

UNIVERSITY OF SOUTHERN CALIFORNIA
STANFORD UNIVERSITY

UNIVERSITY OF TOKYO

UNIVERSITY OF UTAH

WASHINGTON STATE UNIVERSITY

UNIVERSITY OF WASHINGTON

${ }^{*} \quad{ }^{*} \quad{ }^{*}$
AMERICAN MATHEMATICAL SOCIETY
CHEVRON RESEARCH CORPORATION
TRW SYSTEMS
NAVAL WEAPONS CENTER




\section{Pacific Journal of Mathematics}

\section{Vol. 33, No. $1 \quad$ March, 1970}

Mir Maswood Ali, On some extremal simplexes ................... 1

Silvio Aurora, On normed rings with monotone multiplication........... 15

Silvio Aurora, Normed fields which extend normed rings of integers....... 21

John Kelly Beem, Indefinite Minkowski spaces..................... 29

T. F. Bridgland, Trajectory integrals of set valued functions ........... 43

Robert Jay Buck, A generalized Hausdorff dimension for functions and sets ......................................... 69

Vlastimil B. Dlab, A characterization of perfect rings . . . . . . . . . . . . 79

Edward Richard Fadell, Some examples in fixed point theory ............ 89

Michael Benton Freeman, Tangential Cauchy-Riemann equations and uniform approximation ............................. 101

Barry J. Gardner, Torsion classes and pure subgroups ................ 109

Vinod B. Goyal, Bounds for the solution of a certain class of nonlinear

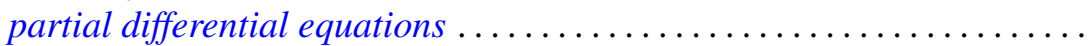

Fu Cheng Hsiang, On C, 1 summability factors of Fourier series at a given

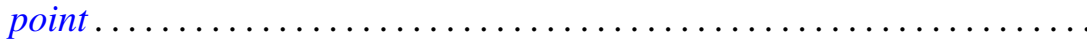

Lawrence Stanislaus Husch, Jr., Homotopy groups of PL-embedding

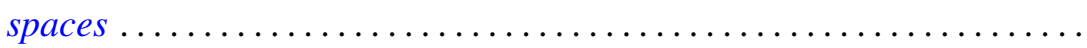

Daniel Ralph Lewis, Integration with respect to vector measures..........

Marion-Josephine Lim, $\mathscr{L}-2$ subspaces of Grassmann product spaces

Stephen J. Pierce, Orthogonal groups of positive definite multilinear functionals

W. J. Pugh and S. M. Shah, On the growth of entire functions of bounded index.

Siddani Bhaskara Rao and Ayyagari Ramachandra Rao, Existence of triconnected graphs with prescribed degrees . . .

Ralph Tyrrell Rockafellar, On the maximal monotonicity of subdifferential

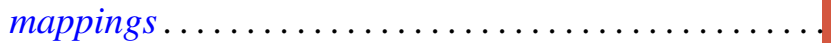

R. Shantaram, Convergence of a sequence of transformations of distribution functions. II ...............................

Julianne Souchek, Rings of analytic functions..............

Ted Joe Suffridge, The principle of subordination applied to functions of several variables...

Wei-lung Ting, On secondary characteristic classes in cobordism

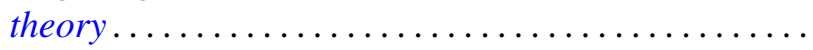

Pak-Ken Wong, Continuous complementors on $B^{*}$-algebras ...

Miyuki Yamada, On a regular semigroup in which the idempotents form a band. 